\title{
Mechanically driven SMR-based NEMS magnetoelectric antennas
}

Xianfeng Liang

Northeastern University

Huaihao Chen

Northeastern University

Neville Sun

Northeastern University

Elizaveta Golubeva

University of Kiel

Cai Müller

Institute for Materials Science, Kiel University

Sushant Mahat

University of Illinois at Urbana-Champaign

Yuyi Wei

Northeastern University

Cunzheng Dong

Northeastern University https://orcid.org/0000-0002-3379-7816

Mohsen Zaeimbashi

Northeastern University

Yifan He

Northeastern University

Yuan Gao

Northeastern University https://orcid.org/0000-0002-2444-1180

Hwaider Lin

Winchester Technologies

David Cahill

University of Illinois https://orcid.org/0000-0001-5969-3460

\section{Mohan Sanghadasa}

U.S. Army Aviation and Missile Research, Development, and Engineering Center Jeffrey McCord

Kiel University

Nian Sun ( $\nabla$ n.sun@northeastern.edu )

Northeastern University 
Article

Keywords:

Posted Date: December 6th, 2021

DOI: https://doi.org/10.21203/rs.3.rs-1118802/v1

License: (c) (i) This work is licensed under a Creative Commons Attribution 4.0 International License. Read Full License 


\section{$1 \quad$ Mechanically driven SMR-based NEMS magnetoelectric antennas}

2 Xianfeng Liang ${ }^{1, *}$, Huaihao Chen ${ }^{1, *}$, Neville Sun ${ }^{1, *}$, Elizaveta Golubeva ${ }^{3}$, Cai Müller ${ }^{3}$, Sushant Mahat ${ }^{5}$, Yuyi Wei ${ }^{1}$,

3 Cunzheng Dong ${ }^{1}$, Mohsen Zaeimbashi ${ }^{1}$, Yifan $\mathrm{He}^{1}$, Yuan $\mathrm{Gao}^{2}$, Hwaider Lin ${ }^{2}$, David G. Cahill ${ }^{5}$, Mohan

4 Sanghadasa ${ }^{6}$, Jeffrey McCord ${ }^{3,4}$ and Nian X. Sun ${ }^{1,2, * *}$

$5 \quad{ }^{1}$ Department of Electrical and Computer Engineering, Northeastern University, Boston, MA 02115, USA

$6 \quad{ }^{2}$ Winchester Technologies, LLC, Burlington, MA 01803, USA

$7 \quad{ }^{3}$ Institute for Materials Science, Kiel University, Kiel, 24143, Germany

$8 \quad{ }^{4}$ KiNSIS - Kiel Nano, Surface and Interface Science, Kiel University, Kiel, 24143, Germany

$9 \quad{ }^{5}$ Department of Materials Science and Engineering, Engineering and Materials Research Laboratory, University of Illinois at 10 Urbana-Champaign, Urbana, IL 61801, USA

$11{ }^{6}$ U.S. Army Combat Capabilities Development Command Aviation \& Missile Center, Redstone Arsenal, AL 35898

$12 *$ Xianfeng Liang, Huaihao Chen and Neville Sun contributed equally to this work.

13 ** Correspondence: n.sun@northeastern.edu; Tel.: +1 (617) 3733351.

14 Abstract-Mechanically driven magnetoelectric (ME) antennas have been demonstrated to be one of the most effective methods to miniaturise antennas compared to state-of-the-art compact antennas. However, the nanoelectromechanical systems (NEMS) ME antennas are fragile due to their suspended thin-film heterostructure, and have very low power handling capabilities. Here we show that solidly mounted resonator (SMR)-based NEMS ME antennas on a Bragg acoustic resonator, which have a circular resonating disk of $200 \mu m$ diameters and operate at $1.75 \mathrm{GHz}$, show a high antenna gain of $-18.8 \mathrm{dBi}$ and $1 \mathrm{~dB}$ compression point (P1dB) of $30.4 \mathrm{dBm}$. Compared to same-size thin-film bulk acoustic resonator (FBAR) ME antennas with a free-standing membrane, the SMR-based antennas are much more structurally stable with $23.3 \mathrm{~dB}$ higher power handling capability and easier fabrication steps. These SMR-based ME antennas are fabricated with processes compatible with complementary metal-oxide-semiconductor (CMOS), exhibiting dramatic size miniaturisation, high power handling, high mechanical robustness, simple fabrication processes, and much higher antenna radiation gain compared to same-size state-of-the-art antennas. 
In the last few decades, explosive growth in miniaturised, low-profile and cost-effective antennas has emerged due to the proliferation of innovative applications such as Internet-of-Things (IoT) devices, 5th generation (5G) wireless systems, millimetre-wave (mmwave) and Terahertz (THz) applications, etc. ${ }^{1,2,3,4}$ There is an insatiable need for small-size and highly efficient antennas. Numerous miniaturisation techniques have been developed to reduce the size of antennas, such as loading materials ${ }^{5}$, lumped elements ${ }^{6}$, geometry designs ${ }^{7,8}$, metamaterials ${ }^{9}$, etc. However, the dimensions of conventional compact antennas are typically larger than $1 / 10$ electromagnetic (EM) wavelength $\lambda_{0}{ }^{10,11}$, which are too large for many current and future technologies, such as implantable and injectable medical devices ${ }^{12,13,14}$.

Since the renaissance of magnetoelectric (ME) composites in the last twenty years ${ }^{15,16,17,18}$, different multiferroic devices ${ }^{19,20,21,22,23}$ have been developed based on the direct (magnetic field control of electric polarisation) or converse (electric field control of magnetisation) ME coupling. The concept of novel ME antennas for antenna size miniaturising has been theoretically proposed ${ }^{24}$ and experimentally demonstrated in the recent past ${ }^{25,26,27,28,29}$. In addition to the investigations on ME antennas that operate at veryhigh frequency (VHF) and ultra-high frequency (UHF) bands, several papers were published on designing mechanical antennas for low frequency (LF) and very-low frequency (VLF) applications based upon a rotating electret or spinning magnet ${ }^{30,31,32,33}$. In 2020, resonance, which has about five orders of magnitude shorter wavelength than that of EM resonance at the same frequency.

Free-standing or released thin-film bulk acoustic resonator (FBAR) ${ }^{35}$ and solidly mounted resonator (SMR) $)^{36}$ are two principal geometric structures for bulk acoustic wave (BAW) devices to confine acoustic energy in the piezoelectric material. An isolating structure with minimised energy dissipation in the neighbouring media is required to build up a high-quality-factor strong standing mechanical resonance. The acoustic isolation of FBAR is realised by the air gap between the resonating plate and the substrate. At the same time, the SMR uses a Bragg acoustic reflector for confining the mechanical energy in the resonating plate. The Bragg acoustic reflector is composed of alternating layers of low and high acoustic impedance materials with controlled thicknesses. The leakage of mechanical energy is prohibited by the reflection of acoustic waves at each interface of the multilayer stack due to the acoustic impedance mismatch. The disadvantages of these released FBARs include (1) limited thermal dissipation capability and, 
therefore, low power handling capability, (2) fragile anchors that lead to fracture and low yield rate, (3) tight control of film stresses developing during fabrication and (4) complex fabrication process steps including the releasing processes. On the other hand, the SMR sits on the Bragg acoustic lattice without any suspended structures and has much higher power handling capabilities with simpler fabrication processes and a higher yield rate. The SMR also has much improved mechanical robustness, which allows it to be easily packaged and integrated with integrated circuits (ICs).

In this paper, we demonstrate a new SMR-based ME antenna to minimise energy loss by returning the vertically propagating energy from the Bragg reflector back to the acoustic resonator. These ME antennas with a 200- $\mu m$-diameter circular disk are designed to have a mechanical vibration mode at UHF $(1.75 \mathrm{GHz})$ operation frequencies. Owing to the Bragg acoustic reflector, the SMR-based ME antennas have an antenna gain of $-18.8 \mathrm{dBi}$, comparable to that of the same-size FBAR ME antennas. The $1 \mathrm{~dB}$ compression point (P1dB) of SMR-based ME antenna was measured to be $30.4 \mathrm{dBm}$, which is $23.3 \mathrm{dBm}$ higher than that of the FBAR ME antennas with the same size. The combined high power handling capability, high antenna gain, easy fabrication process makes these SMR ME antennas great candidate antennas for different wireless systems.

\section{Magnetoelectric composites of the SMR-based ME antenna}

The quality of the Bragg reflector sandwiched between the resonator and substrate plays an important role in producing high-class mechanical antennas. The acoustic properties of a variety of materials normally used for modelling and developing Bragg reflectors in SMR devices are measured and summarised (see Supplementary Table 1 and Supplementary Fig. 2). In Fig. 1a and b, the crosssectional scanning electron microscopy (SEM) image and optical image of the developed ME antenna are presented. The excellent film quality of the Bragg reflector and the ME composite can be seen from these pictures. The signal (S) pad and ground ring (G) structure are used to feed the RF signal into the ME antenna.

The microstructure, crystal orientation, and piezoelectric properties of $\mathrm{ZnO}$ thin films are greatly influenced by the sputtering conditions. We investigated the effects of RF plasma power, sputtering gas pressure, oxygen $\left(\mathrm{O}_{2}\right)$ flux density and temperature on the crystal orientation of $\mathrm{ZnO}$ films to obtain optimal film properties. During the development of $\mathrm{ZnO}$ films, all samples were deposited on the Si substrate and evaluated by x-ray diffraction (XRD) and the full width at half maximum (FWHM) of the rocking curve analysed at the (002) peak. The (002) peak of $\mathrm{ZnO}$ and smallest FWHM is around $34.4^{\circ}$ and $2.29^{\circ}$, respectively, which indicates good 
film quality with high c-axis orientation (see Fig. 1c-h). All other samples are amorphous without any ZnO XRD peak that were discovered. The intrinsic stress is one of the most important characteristics for thin-film free-standing membrane ME devices. However, stress optimisation was not as important as the longer necessary due to the inherent durability of SMR devices.

The magnetic properties of $\mathrm{FeGaB} / \mathrm{SiO}_{2}$ multilayers have been characterised using vibration sample magnetometry (VSM), ferromagnetic resonance (FMR) spectroscopy, and magneto optic Kerr effect (MOKE) imaging ${ }^{37}$. The results for both a reference sample with $5 \mathrm{~mm} \times 5 \mathrm{~mm}$ full film deposited directly on Si substrate and a patterned device with the magnetic stack deposited on the $\mathrm{ZnO}$ film are displayed in Fig. 2. All magnetic films were sputtered onto the substrates under an in-situ magnetic field of $H_{\text {in-situ }}$ of $\sim 2.4 \times 10^{4} \mathrm{~A} / \mathrm{m}$ to induce a well-defined in-plane uniaxial magnetic anisotropy (UMA). Fig. 2a and d illustrates the coordinate systems for in-plane measurements of the reference film and patterned devices. 0 degree corresponds to the anchor direction of ME antennas.

To evaluate the magnetic anisotropy of $\mathrm{FeGaB} / \mathrm{SiO}_{2}$ multilayers, room temperature magnetic magnetisation loops are measured along the 0 and 90 degrees axes and shown in Fig. $2 \mathrm{~b}$ and e for the full film and the SMR device. A clear presence of a well-defined inplane uniaxial magnetic anisotropy (UMA) is seen in the reference film (Fig. 2b). A small coercive field of $112 \mathrm{~A} / \mathrm{m}$ with an estimated effective in-plane anisotropy field $H_{\mathrm{k}}$ of $\sim 1440 \mathrm{~A} / \mathrm{m}$ are observed. The obtained soft magnetic properties are significant for achieving large piezomagnetic coefficients. Nevertheless, while the magnetic multilayers were directly deposited on $\mathrm{ZnO}$ films and patterned for ME antennas, the magnetic anisotropy field decreases to $H_{\mathrm{k}} \sim 1120 \mathrm{~A} / \mathrm{m}$ (Fig. 2e), but also displaying soft magnetic properties. In order to identify the orientation of the effective magnetic anisotropy axis and to obtain more quantitative information, the angular dependence of the in-plane FMR fields was measured. A clear twofold distribution can be seen in Fig. $2 \mathrm{c}$ and $\mathrm{f}$ from the FMR data for both reference films and ME antennas, which is a characteristic feature of UMA. The angle of the axis and strength of the anisotropy field $H_{\mathrm{k}}$ is determined by fitting the obtained FMR field values $H_{\mathrm{r}}$ with the following function: $H_{r}=2 H_{K}$ * $\sin ^{2}(\varphi-\theta)+H_{0}$, where $H_{K}$ is the anisotropy field, $\varphi$ is the angle of the applied DC magnetic field, $\theta$ is the tilt of the UMA axis relative to the 90 degrees axis, and $H_{0}$ is a constant field defined by the chosen fixed resonance frequency. The obtained magnitude of $H_{K}$ and the tilt of the effective magnetic anisotropy axis is $1920 \mathrm{~A} / \mathrm{m}$ and 12.8 degrees for the reference film and $720 \mathrm{~A} / \mathrm{m}$ and 11.3 degrees for the ME antenna. 
The source of UMA can be in principle attributed to magnetocrystalline anisotropy, substrate surface topography, magnetic field induced anisotropy and stress induced anisotropy ${ }^{38}$. Due to the amorphous structure of the thick seed layer and smooth substrate surface, we assume only contributions from the induced anisotropies to the UMA. Wang et al. ${ }^{38}$ demonstrated the stress-induced UMA in FeCoC films. However, the deposited film stress was not analysed. In the current work, a detailed investigation on the anisotropic stress of FeGaB films is carried out and shown in Supplementary Fig. 1, which suggests an additional stress-induced UMA from the initial layers during growth.

The general magnetic anisotropy alignment is confirmed by spatially resolved magnetic analysis of an identical device (Fig 2 g-h and Supplementary Fig. 6). The magnetic domains walls tend to align roughly along the $\sim 90$ degrees axis. Despite the exhibited roughness of the samples, the magnetic anisotropy alignment is still determining the magnetic microstructure. Deviations of the domain wall alignment are related to the laminated structure of the multilayer. This and the observed wide magnetic domain walls, an indication of low magnetic film coupling, confirm the quality of the film structure. Noticeable is the curving of the magnetisation from the edges are caused by stress relaxation ${ }^{39}$, leading to a stress-induced anisotropy contribution aligned parallel to edges. This indicates tensile stress for the positive magnetostrictive films. This further transfers into a change of the magnetic anisotropy strength in the more central region of the disc shaped device, leading to a spatially varying anisotropy strength alteration. Another factor that plays a role is the change of the magnetic properties in the anchor region, visible from the analysis of the magnetisation process (Supplementary Fig. 6). Overall, the tilt of magnetic anisotropy and the change of integral anisotropy strength of the patterned device structure relative to the full film can be related to spatially varying stress induced anisotropy contributions and the effect of roughness on the coercivity of the sample is not significant in this case.

\section{Design and simulations of the SMR-based ME antenna}

Prior to fabricating SMR devices, various simulation methods were implemented to design and optimise the performance of SMRbased ME antennas with a focus on the Bragg reflector and ME composites. The first analysis was carried out by using the 1D Mason's model to estimate the reflection coefficient frequency response of a carefully arranged Bragg reflector ${ }^{40,41,42}$. The equivalent circuit model of the SMR-based ME antenna analysed by the Mason's model and based on the transmission line theory is shown in Fig. 3a. The load acoustic impedance decreases while the total number of Bragg reflector layers (n) increases. Therefore, a large value of $\mathrm{n}$ is desired for the resonator structure. Typically, three pairs of low/high acoustic impedance layers $(\mathrm{n}=6)$ are chosen for SMRs. 
In order to correlate the working frequency bandwidth of the Bragg reflector with the resonant frequency of the piezoelectric resonator, the thickness of each layer is decided by the equation: $d_{\text {acoustic }}=v_{\text {acoustic }} / 4 f$, where $d_{\text {acoustic }}$ and $v_{\text {acoustic }}$ are the thickness and acoustic velocity of the Bragg reflector layers, respectively. In this work, the working frequency of SMR-based ME antenna was not specified. Therefore, the thickness of each layer was not exactly a quarter wavelength of the acoustic wave. The simulated reflection coefficient of the designed Bragg reflector consisting of three pairs of $\mathrm{SiO}_{2} / \mathrm{W}$ layers was calculated. The results of which are shown in Fig. 3b. The total reflection seen from the resonant structure is formed over a wide frequency range from 1 to $3 \mathrm{GHz}$. The influence on the reflection coefficient with varying number of periods is also exploited and displayed in Fig. 3b. As more periods are applied, more energy is reflected from Bragg acoustic lattice, and so the frequency band widens. The calculation results for more periods of $\mathrm{SiO}_{2} / \mathrm{W}$ layers are presented in Supplementary Fig. 3, which illustrates minimal improvement in the reflection coefficient when the number of periods exceeds three.

To achieve a frequency domain analysis of the admittance spectrum, a 2D model of the ME antenna was developed, and the coupling between electrical potential and mechanical displacement in the antenna was simulated by the finite element method (FEM) in COMSOL Multiphysics v5.142. The magnitude of the total displacement profile of the longitudinal waves and the standing wave amplitude as a function of depth of the designed ME antenna at the electromechanical resonant frequency of $1.7 \mathrm{GHz}$ are presented in Fig. $3 \mathrm{c}$ and d. The 2D strain assumption is used in this model and the thickness of each layer is defined with the value as shown in Supplementary Table 1. The developed Bragg reflector is shown with optimised performance as the acoustic wave energy is well confined within the ME composites with little energy dissipating into the substrate. The return loss curve of the ME antenna is plotted in Fig. 3e, which is achieved by the frequency domain analysis with the 2D FEM COMSOL model. The thicknesses of each individual layer in the Bragg reflector and ME composites determines the working frequency of SMR antenna. As explained previously, the performance of SMRs can be improved by adjusting the thickness of the Bragg reflector layer to be a quarter wavelength of the acoustic wave.

\section{Mechanical ME antenna performance}

The antenna gain was characterised in an anechoic chamber by utilising a calibrated linear polarisation standard horn antenna. As shown in Fig. $4 \mathrm{a}$, the return loss curve $\left(\mathrm{S}_{11}\right)$, receiving $\left(\mathrm{S}_{12}\right)$ and transmitting behaviour $\left(\mathrm{S}_{21}\right)$ of the SMR antenna with a resonant frequency of $1.75 \mathrm{GHz}$ and antenna gain of $-18.8 \mathrm{dBi}$ are presented. The $\mathrm{S}_{12}$ and $\mathrm{S}_{21}$ curves overlap with each other. The 
electromechanical resonance frequency, which is defined by the thickness of the ME disk and is validated by the 2D COMSOL model, is expressed as:

$$
f_{0} \propto \frac{1}{2 T} \sqrt{\frac{E}{\rho}}
$$

where $\mathrm{T}$ is the thickness of the ME disk, $\mathrm{E}$ and $\rho$ are the equivalent Young's modulus and equivalent density of the resonator, respectively. In Fig. 4b, the schematics and the fitting parameters for the modified Butterworth Van Dyke (MBVD) model with electrical and equivalent mechanical components of SMR antenna are presented. The electromechanical coupling coefficient $\left(k_{t}^{2}\right)$ and quality factor $(\mathrm{Q})$ are calculated as $1.0 \%$ and 95 , respectively. The MBVD fitting curve for return loss $\mathrm{S}_{11}$ is plotted in Fig. 4c and matches the measurements well. Compared to the released FBAR ME antenna, the SMR antenna has a $10 \mathrm{dBi}$ higher gain. The gain enhancement of the SMR antenna is attributed to the Bragg reflector helping to confine more acoustic energy in the ME films allowing for greater amplitude in EM waves.

A high linearity is always wanted for the components in the RF systems such as filters, amplifiers and antennas. This is desired because there are numerous different bands, and they have to be protected from any undesired signals. Furthermore, nonlinearity of the devices can undesirably degrade the performance of the system. It has been reported that the acoustic resonators exhibit a nonlinear behaviour at high power levels $\mathrm{s}^{43,44,45}$. Since the power density and temperature play significant roles in controlling the nonlinearity, various methods such as the device structure, area and materials etc. are used to improve the linearity of acoustic resonators ${ }^{46}$.

The power handling capability of the FBAR and SMR antennas are characterised by the power sweep results shown in Fig. 4f. The $\mathrm{P} 1 \mathrm{~dB}$ is acquired by measuring the $\mathrm{S}_{21}$ curves as a function of input power. The FBAR antenna has a P1dB of $7.1 \mathrm{dBm}$ while the P1dB of SMR antenna is $30.4 \mathrm{dBm}$, which shows a better power handling capability of the SMR antenna. The $\mathrm{S}_{11}$ curves of the FBAR and SMR antennas at different input power levels are measured and shown in Fig. $4 \mathrm{~d}$ and e. The resonance peak of the FBAR antenna shifts to lower frequency at high input power while the SMR antenna remains unchanged, which means the FBAR antenna starts going into nonlinear region. This can be explained by the self-heating effects at high power levels. As the input power extremely increases, the high power density leads to self-heating of the resonator and higher temperature. Compared to the free-standing FBAR membrane structure, the SMR antenna with acoustic Bragg reflector stacks has a larger thermal conductivity. This compensation effect results in a better power handling capability. The comparison of performance metrics between the FBAR and SMR antennas are listed in Table 1. 
2 The radiation characteristics of the SMR-based ME antennas were tested in a far-field configuration with a distance of $0.76 \mathrm{~m}$ between the SMR antenna and the horn antenna. The active radiative element of the SMR antenna is defined by the $\mathrm{ZnO} / \mathrm{FeGaB} \mathrm{ME}$ heterostructure disk with a diameter of $200 \mu \mathrm{m}$. Owing to the small size of the SMR antenna and the limitations of the probe station, we were only able to measure the in-plane radiation pattern 180 degrees around the SMR device. The schematics and measured radiation pattern are shown in Fig. 5a. A dipole-like radiation pattern is assumed according to the symmetry of the SMR antenna. The radiation pattern shows an approximate 7-degree shift from the centre of the horn antenna due to the in-plane tilt of the UMA, as shown in Fig. 2. The maximum gain is located along the tilted direction of the anchor length (the 7-degree point as shown in Fig. 5e), which is the hard axis (H.A.) of the magnetostrictive FeGaB film. At 7 degrees, the RF magnetic field component of EM wave is parallel to the magnetisation of the magnetostrictive layer, which results in maximum coupling efficiency between the SMR antenna and EM wave to achieve maximum gain. When in-plane radiation is measured at 97 degrees from the centre, the antenna gain approaches its null value because the RF magnetic field is perpendicular to the magnetisation.

The polarisation behaviours of SMR ME antennas were measured by rotating the standard horn antenna along three major axes, as shown in Fig. 5b-d. In the schematic representations of Fig. 5, the horn antenna was rotated along the out-of-plane axis (Fig. 5b), inplane axis perpendicular to the anchor direction (Fig. 5c) and in-plane axis along the anchor direction (Fig. 5d). The sinusoidal wave along $0^{\circ}$ and $180^{\circ}$ in all the schematics indicates the RF H-field component of the EM waves from the horn antenna. The normalised gain plot in Fig. 5f-h show a similar shape of bifold symmetry, which results from the in-plane uniaxial magnetic anisotropy of the $\mathrm{FeGaB} / \mathrm{ZnO}$ multilayer in the resonating disk of the SMR antenna.

As presented in Fig. 5f, the highest gain of the SMR antenna is achieved along the easy axis (E.A.) when the $\mathrm{H}_{\mathrm{rf}}$ is parallel to the E.A. direction. The lowest gain is measured tilted away from the 0-degree position when the $\mathrm{H}_{\mathrm{rf}}$ is parallel to the H.A. direction. The other two polarisation charts in Fig. 5g and h show a similar behaviour where the maximum gain is obtained along the E.A. direction of magnetic anisotropy. This is because the strongest coupling between $\mathrm{H}_{\mathrm{rf}}$ and SMR ME antenna is achieved when $\mathrm{H}_{\mathrm{rf}}$ is parallel to the magnetic easy axis in the ME disk. The measured results in Fig. 5 demonstrate that the strong coupling between the H-field component of $\mathrm{EM}$ waves and the magnetostrictive phase of $\mathrm{FeGaB} / \mathrm{ZnO}$ heterostructures in SMR antenna will ultimately improve $\mathrm{ME}$ antenna gain. 


\section{Conclusion}

3 The latest mechanically driven antennas have pushed the boundaries of antenna miniaturisation to micrometre dimensions starting with the free-standing membrane FBAR design. The presented SMR structures for ME antennas not only improve upon the antenna performance metrics but also simplifies the process with better device structural integrity and removing the extra packaging steps required for device protection. We have demonstrated a working micro-sized ME antenna that takes advantage of a solidly mounted resonator to confine energy in the magnetostrictive/piezoelectric heterostructure showing improvements in antenna radiation. The SMR ME antenna, with an overall dimension of $700 \mu m \times 700 \mu m(\mathrm{~L} \times \mathrm{W})$, was designed and optimised with the 1D Mason model and a 2D COMSOL FEM simulation to operate at a resonant frequency of $1.75 \mathrm{GHz}$ and experimentally demonstrated a gain of -18.8 dBi. Further improvements can be made by increasing the SMR quality factor and optimising the impedance matching with the signal feedline to the resonator.

The SMR ME antennas have demonstrated more robust features than its FBAR freestanding membrane counterparts. Due to its small size, high operating frequency, high sensitivity, structural stability, semiconductor processing integration, and good power handling capability, it is the ideal device for small-size microwave antennas and remote wireless sensing applications used for compact UAVs (unmanned aerial vehicles), bio-implantable antennas, wearable antennas, IoT (internet of things), NFC (near field communication), RFID (radio frequency identification), satellites, and many more existing and to be envisioned applications.

\section{Methods}

Methods and any associated references are available in the online version of the paper.

Received: ; Accepted: ; Published online:

\section{References}

1. He Y, Pan Z, Cheng X, He Y, Qiao J, Tentzeris MM. A novel dual-band, dual-polarized, miniaturized and low-profile base station antenna. IEEE Transactions on Antennas and Propagation 63, 5399-5408 (2015).

2. Lizzi L, Ferrero F, Monin P, Danchesi C, Boudaud S. Design of miniature antennas for IoT applications. In: 2016 IEEE Sixth International Conference on Communications and Electronics (ICCE)). IEEE (2016).

3. Hong W, Baek K-H, Lee Y, Kim Y, Ko S-T. Study and prototyping of practically large-scale mmWave antenna systems for 5G cellular devices. IEEE Communications Magazine 52, 63-69 (2014). 
4. Hong W, et al. Multibeam antenna technologies for 5G wireless communications. IEEE Transactions on Antennas and Propagation 65, 6231-6249 (2017).

5. Buell K, Mosallaei $\mathrm{H}$, Sarabandi K. A substrate for small patch antennas providing tunable miniaturization factors. IEEE Transactions on Microwave Theory and Techniques 54, 135-146 (2006).

6. Skrivervik AK, Zurcher J-F, Staub O, Mosig J. PCS antenna design: The challenge of miniaturization. IEEE Antennas and propagation Magazine 43, 12-27 (2001).

7. Gianvittorio JP, Rahmat-Samii Y. Fractal antennas: A novel antenna miniaturization technique, and applications. IEEE Antennas and Propagation magazine 44, 20-36 (2002).

8. Werner DH, Ganguly S. An overview of fractal antenna engineering research. IEEE Antennas and propagation Magazine 45, 38-57 (2003).

9. Dong Y, Itoh T. Metamaterial-based antennas. Proceedings of the IEEE 100, 2271-2285 (2012).

10. Wheeler HA. Fundamental limitations of small antennas. Proceedings of the IRE 35, 1479-1484 (1947).

11. Volakis J, Chen C-C, Fujimoto K. Small antennas: miniaturization techniques \& applications. McGraw Hill Professional (2009).

12. Kiourti A, Nikita KS. A review of implantable patch antennas for biomedical telemetry: Challenges and solutions [wireless corner]. IEEE Antennas and Propagation Magazine 54, 210-228 (2012).

13. Scarpello ML, et al. Design of an implantable slot dipole conformal flexible antenna for biomedical applications. IEEE Transactions on Antennas and Propagation 59, 3556-3564 (2011).

14. Kiourti A, Nikita KS. A review of in-body biotelemetry devices: implantables, ingestibles, and injectables. IEEE Transactions on Biomedical Engineering 64, 1422-1430 (2017).

15. Nan C-W, Bichurin M, Dong S, Viehland D, Srinivasan G. Multiferroic magnetoelectric composites: Historical perspective, status, and future directions. Journal of applied physics 103, 1 (2008).

16. Srinivasan G. Magnetoelectric composites. Annual Review of Materials Research 40, 153-178 (2010).

17. Sun NX, Srinivasan G. Voltage control of magnetism in multiferroic heterostructures and devices. In: Spin). World Scientific (2012).

18. Liang X, Chen H, Sun NX. Magnetoelectric materials and devices. APL Materials 9, 041114 (2021).

19. Li M, et al. Highly sensitive DC magnetic field sensor based on nonlinear ME effect. IEEE sensors letters 1, 1-4 (2017).

20. Tu C, Dong C, Chu Z, Chen H, Liang X, Sun NX. A passive isolator realized by magnetoelectric laminate composites. Applied Physics Letters 113, 262904 (2018).

21. Chu Z, et al. A low-power and high-sensitivity magnetic field sensor based on converse magnetoelectric effect. Applied Physics Letters 115, 162901 (2019).

22. Chen H, et al. Integrated Tunable Magnetoelectric RF Inductors. IEEE Transactions on Microwave Theory and Techniques, (2020).

23. Liang X, et al. A Review of Thin-Film Magnetoelastic Materials for Magnetoelectric Applications. Sensors 20, 1532 (2020).

24. Yao Z, Wang YE, Keller S, Carman GP. Bulk acoustic wave-mediated multiferroic antennas: Architecture and performance bound. IEEE Transactions on Antennas and Propagation 63, 3335-3344 (2015). 
25. Nan T, et al. Acoustically actuated ultra-compact NEMS magnetoelectric antennas. Nature Communications 8, 296 (2017).

26. Liang X, Chen H, Sun N, Lin H, Sun NX. Novel Acoustically Actuated Magnetoelectric Antennas. In: 2018 IEEE International Symposium on Antennas and Propagation \& USNC/URSI National Radio Science Meeting). IEEE (2018).

27. Chen H, et al. Ultra-compact mechanical antennas. Applied Physics Letters 117, 170501 (2020).

28. Chen H, Liang X, Sun N, Sun N-X, Lin H, Gao Y. An Ultra-Compact ME Antenna Design for Implantable Wireless Communication. In: 2020 IEEE International Symposium on Antennas and Propagation and North American Radio Science Meeting). IEEE (2020).

29. Zaeimbashi M, et al. Ultra-compact dual-band smart NEMS magnetoelectric antennas for simultaneous wireless energy harvesting and magnetic field sensing. Nature Communications 12, 1-11 (2021).

30. Bickford JA, McNabb RS, Ward PA, Freeman DK, Weinberg MS. Low frequency mechanical antennas: Electrically short transmitters from mechanically-actuated dielectrics. In: 2017 IEEE International Symposium on Antennas and Propagation \& USNC/URSI National Radio Science Meeting). IEEE (2017).

31. Selvin S, Prasad MS, Huang Y, Wang E. Spinning magnet antenna for VLF transmitting. In: 2017 IEEE International Symposium on Antennas and Propagation \& USNC/URSI National Radio Science Meeting). IEEE (2017).

32. Burch HC, Garraud A, Mitchell MF, Moore RC, Arnold DP. Experimental generation of ELF radio signals using a rotating magnet. IEEE Transactions on Antennas and Propagation 66, 6265-6272 (2018).

33. Bickford JA, Duwel AE, Weinberg MS, McNabb RS, Freeman DK, Ward PA. Performance of electrically small conventional and mechanical antennas. IEEE Transactions on Antennas and Propagation 67, 2209-2223 (2019).

34. Dong C, et al. A Portable Very Low Frequency (VLF) Communication System Based on Acoustically Actuated Magnetoelectric Antennas. IEEE Antennas and Wireless Propagation Letters, (2020).

35. Ruby RC, Bradley P, Oshmyansky Y, Chien A, Larson J. Thin film bulk wave acoustic resonators (FBAR) for wireless applications. In: 2001 IEEE Ultrasonics Symposium. Proceedings. An International Symposium (Cat. No. 01CH37263)). $\operatorname{IEEE}(2001)$.

36. Newell W. Face-mounted piezoelectric resonators. Proceedings of the IEEE 53, 575-581 (1965).

37. McCord J. Progress in magnetic domain observation by advanced magneto-optical microscopy. Journal of Physics D: Applied Physics 48, 333001 (2015).

38. Wang J, et al. Magnetostriction, Soft Magnetism, and Microwave Properties in Co-Fe-C Alloy Films. Physical Review Applied 12, 034011 (2019).

39. McCord J. Irregular domain patterns in structured magnetic thick films. Journal of applied physics 95, 6855-6857 (2004).

40. Nakamura K, Kanbara H. Theoretical analysis of a piezoelectric thin film resonator with acoustic quarter-wave multilayers. In: Proceedings of the 1998 IEEE International Frequency Control Symposium (Cat. No. 98CH36165)). IEEE (1998).

41. Cannatà D, et al. Nerve agent simulant detection by solidly mounted resonators (SMRs) polymer coated using laser induced forward transfer (LIFT) technique. Sensors and Actuators B: Chemical 173, 32-39 (2012).

42. Villa-López FH, Rughoobur G, Thomas S, Flewitt AJ, Cole M, Gardner JW. Design and modelling of solidly mounted resonators for low-cost particle sensing. Measurement Science and Technology 27, 025101 (2015).

43. Nosek J. Drive level dependence of the resonant frequency in BAW quartz resonators and his modeling. IEEE transactions on ultrasonics, ferroelectrics, and frequency control 46, 823-829 (1999). 
44. Kaajakari V, Mattila T, Oja A, Seppa H. Nonlinear limits for single-crystal silicon microresonators. Journal of Microelectromechanical systems 13, 715-724 (2004).

45. Aigner R, Huynh N-H, Handtmann M, Marksteiner S. Behavior of BAW devices at high power levels. Proceedings of IEEE IMS-MTT-S, (2005).

46. Schmidhammer E. Nonlinear effects in acousto-electric devices. In: 2008 4th European Conference on Circuits and Systems for Communications). IEEE (2008).

\section{Methods}

Materials decision. The acoustic impedance of various materials is the key point for creating effective Bragg reflectors used for $\mathrm{ME}$ antennas. Silicon $(\mathrm{Si})$, silicon dioxide $\left(\mathrm{SiO}_{2}\right)$ and aluminium $(\mathrm{Al})$ are common low impedance materials for Bragg reflectors while platinum $(\mathrm{Pt})$, tungsten $(\mathrm{W})$ and iridium (Ir) are introduced as high impedance materials. In this work, we determined that employing sputtered $\mathrm{SiO}_{2} / \mathrm{W}$ as the low/high acoustic impedance materials to construct the Bragg reflector will provide the best acoustic impedance ratio along with a cost-effective fabrication process. In order to achieve strong ME coupling in the magnetostrictive/piezoelectric ME composites, ferromagnetic/ferroelectric materials with large piezomagnetic/piezoelectric coefficients are desired. Excellent magnetic softness and magnetostrictive behaviours have been realised in FeGa-based thin films ${ }^{47}$, ${ }^{48}$, which have already been applied in different $\mathrm{RF} /$ microwave tuneable devices $^{22,25}$. Aluminium nitride (AlN) and zinc oxide ( $\mathrm{ZnO}$ ) are two of the most popular piezoelectric materials for FBAR devices, among which, AlN has been chosen as the material for commercial BAW filters that operates at $1-2 \mathrm{GHz}$ because of its quality factor (Q). However, $\mathrm{ZnO}$ has larger $K_{t}^{2}$ and wider bandwidth than AIN due to its higher piezoelectric coefficient $\left(\mathrm{d}_{33}\right)$. Moreover, the deposition, control of texture, and stoichiometry of $\mathrm{ZnO}$ is much easier compared with that for $\mathrm{AlN}^{49,50} . \mathrm{ZnO}$ films with highly c-axis-preferred orientation are essential for realising high-quality SMR-type ME antennas. To fabricate SMR-based ME antennas, $\mathrm{SiO}_{2} / \mathrm{W}$ as the low/high impedance Bragg reflector and $\mathrm{FeGaB} / \mathrm{ZnO}$ as the magnetostrictive/piezoelectric $\mathrm{ME}$ composite were carefully designed and deposited by $\mathrm{RF}$ magnetron sputtering. Platinum $(\mathrm{Pt})$ and gold $(\mathrm{Au})$ were chosen as the bottom and top electrodes, respectively, due to the benefits for growing highly c-axis-textured $\mathrm{ZnO}$ thin films and decent conductivity.

Thin films deposition. All thin-film materials were prepared in a magnetron sputtering system at the Argon (Ar) flux density of 15 sccm with a base pressure of $\sim 1 \times 10^{-7}$ Torr. The oxides including $\mathrm{SiO}_{2}$ and $\mathrm{ZnO}$ were deposited by reactive $\mathrm{RF}$ sputtering and other metallic thin films involving $\mathrm{W}, \mathrm{FeGaB}, \mathrm{Pt}$, and Au were DC sputtered. All materials were deposited at room temperature except $\mathrm{ZnO}$ layer, which was deposited at $450{ }^{\circ} \mathrm{C}$ to achieve a highly c-axis-orientated structure. For the Bragg reflector with structure of $\left[\mathrm{W}(531 \mathrm{~nm}) / \mathrm{SiO}_{2}(543 \mathrm{~nm})\right]_{3}$, W layers were sputtered at a plasma power of $50 \mathrm{~W}$ and an Ar atmosphere of $4.5 \mathrm{mTorr}$; 
$\mathrm{SiO}_{2}$ layers were deposited by reactive $\mathrm{RF}$ sputtering from a Si target with a 10:1 $\mathrm{Ar} / \mathrm{O}_{2}$ gas mixture at a plasma power of $75 \mathrm{~W}$ and

a sputtering pressure of $6 \mathrm{mTorr}$. For the piezoelectric/magnetostrictive ME composite, the piezoelectric ZnO layer was prepared by reactive RF sputtering from a $\mathrm{Zn}$ target with a 3:1 $\mathrm{Ar} / \mathrm{O}_{2}$ gas mixture at a plasma power of $150 \mathrm{~W}$ and a sputtering pressure of 3 mTorr; the magnetostrictive multilayer with the structure of $\left[\mathrm{FeGaB}(85 \mathrm{~nm}) / \mathrm{SiO}_{2}(5 \mathrm{~nm})\right]_{10}$ was deposited on a $10 \mathrm{~nm} \mathrm{Cr}$ seed layer at a plasma power of $50 \mathrm{~W}$ and a sputtering pressure of $3 \mathrm{mTorr}$. The $\mathrm{FeGaB}$ and $\mathrm{SiO}_{2}$ layers were sputtered from a $\left(\mathrm{Fe}_{80} \mathrm{Ga}_{20}\right)_{88} \mathrm{~B}_{12}$ (DC sputtering) target and a $\mathrm{SiO}_{2}$ (RF sputtering) target, respectively. $\mathrm{Ti}(5 \mathrm{~nm}) / \mathrm{Pt}(50 \mathrm{~nm})$ and $\mathrm{Cr}(5 \mathrm{~nm}) / \mathrm{Au}(100 \mathrm{~nm})$ electrodes were prepared at $30 \mathrm{~W}$ and 3 mTorr. X-ray reflectivity method was used to calibrate the deposition rates.

Device fabrication. A high resistivity $(>10000 \Omega \cdot \mathrm{cm}) \mathrm{Si}$ wafer was chosen to begin the process. The Bragg reflector consisting of three pairs of $\mathrm{W} / \mathrm{SiO}_{2}$ layers was sputter-deposited in one run with $\mathrm{RF}$ reactive magnetron sputtering for $\mathrm{SiO}_{2}$ and $\mathrm{DC}$ sputtering for W in our PVD system. A 50-nm-thick Pt film was sputter-deposited and patterned by lift-off on top of the Bragg reflector to establish the bottom electrodes. Then, the $\mathrm{ZnO}$ film was sputter-deposited by RF reactive sputtering with $\mathrm{Zn}$ target and high-purity $\mathrm{O}_{2}$ gas and vias were etched by Hydrochloric $(\mathrm{HCl})$ acid to access the bottom electrodes. After that, a 100-nm-thick Au film was sputtered and patterned by lift-off to form the top ground electrode. Finally, the $\mathrm{FeGaB} / \mathrm{SiO}_{2}$ multilayer layer was deposited by a magnetron sputtering and patterned by lift-off. A $2.4 \times 10^{4} \mathrm{~A} / \mathrm{m}$ in-situ magnetic field bias perpendicular to the anchor direction of the device SMR antenna with a resonator diameter of $200 \mu \mathrm{m}$ was characterised.

FMR and MOKE measurements. An electron paramagnetic resonance spectrometer operating at X-band (9.6 GHz) with a TE 102 microwave cavity was utilised to characterise the in-plane angular dependence of FMR field for the reference full film and patterned device. The DC magnetic field was applied in the film plane and perpendicular to the magnetic component of the microwave field. Magnetooptical Kerr effect (MOKE) microscopy ${ }^{39}$ in the longitudinal mode was employed for the analysis of the magnetisation and magnetic domain behaviour in the SMR-based ME devices. An illumination wavelength of $460 \mathrm{~nm}$ was used for the MOKE imaging in combination with a differential imaging technique for low magnetooptical signal detection. The in-plane magnetic sensitivity was varied for obtaining complementary magnetic domain and magnetic domain wall magnetooptical contrast. The in-plane applied magnetic field direction is freely rotatable and was varied during the experiments. 
2 S parameters measurement. The SMR antennas and horn antenna are connected to port 1 and 2 of a vector network analyser

3 (VNA) (Agilent E8364A) in order to measure the return loss and transmission curves. Before measuring the devices, a short-open-

4 load-through calibration was performed. For various setting parameters of the VNA, the output power was $-12 \mathrm{dBm}$ and the

5 intermediate frequency (IF) was set to $500 \mathrm{~Hz}$. A home-made 3D-printed plastic RF probe station with configured ground-signal-

6 ground probes was utilised to test the devices. All SMR antennas are fabricated and characterised in a $15 \times 15 \mathrm{~mm}^{2}$ chip due to their $7 \quad$ small size of $0.7 \times 0.7 \mathrm{~mm}^{2}$.

9 1D Mason model calculation. The equivalent circuit structure (Fig. 3a) contains the Si substrate, the Bragg reflector (3 pairs of 10 alternating $\mathrm{W} / \mathrm{SiO}_{2}$ layers), and the piezoelectric $\mathrm{ZnO}$ film sandwiched between the bottom Pt electrode and the magnetostrictive $11 \mathrm{FeGaB} / \mathrm{SiO}_{2}$ multilayers. As noted in the equivalent circuit, $\mathrm{Z}$ is the acoustic impedance, $\mathrm{k}$ is the propagation constant, and $\mathrm{d}$ is the layer thickness. Subscripts m, p, e, l, h and s denote the magnetostrictive, piezoelectric, bottom electrode, low acoustic impedance, high acoustic impedance and substrate layers, respectively. Additionally, $\mathrm{C}_{0}$ is the static capacitance of the piezoelectric resonator; A represents the electrode area; $\varepsilon_{33}$ and $e_{33}$ are the dielectric and piezoelectric constants of the piezoelectric layer; $Y_{\text {in }}$ is the input admittance of the electric port. The effective load acoustic impedance to the resonator seen from the interface between the Bragg reflector and bottom electrode is:

$$
Z_{\text {load }}=\left\{\begin{array}{lc}
\left(\frac{z_{l}}{z_{h}}\right)^{n-1}\left(\frac{z_{l}^{2}}{z_{s}}\right) & (n: \text { odd }) \\
\left(\frac{z_{l}}{z_{h}}\right)^{n} Z_{s} & (n: \text { even })
\end{array}\right.
$$

where $\mathrm{n}$ is the total number of Bragg reflector layers.

Finite element analysis of electromechanical properties. To analyse the resonance mode and displacement of the SMR device, piezoelectric module in COMSOL Multiphysics v5.1 was used for the electromechanical simulation ${ }^{25}$. The modules of solid mechanics and electrostatics were carried out to investigate its performance. The simulation was performed at the frequency domain in a 2D geometry. For the piezoelectric $\mathrm{ZnO}$ layer, a small signal behaviour can be described by constitutive relations in the PZD modules. The fixed constraint condition is applied to the edges of the structure and all other boundaries are set as free. The bottom Pt 
1 A mapped mesh with different distributions is used to form the meshing of SMR-based ME antenna. More details of the analysis can

2 be found in reference [26].

4 MBVD model fitting. MBVD model as shown in Fig. $4 \mathrm{~b}$ is utilised to extract the electromechanical parameters: $k_{t}^{2}$ and Q-factor. $k_{t}^{2}$ denotes the conversion efficiency between electrical and acoustic energy; Q-factor represents the ratio of energy stored and dissipated in the resonator. $R_{s}$ represents the ohmic losses in the electrodes; $R_{m}, L_{m}$, and $C_{m}$ are the motional resistance, inductance and capacitance, respectively; $\mathrm{R}_{0}$ and $\mathrm{C}_{0}$ are related to the dielectric losses of the piezoelectric layer. The $k_{t}^{2}$ and Q-factor are defined as:

$$
\begin{aligned}
& k_{t}^{2}=\frac{\pi^{2} C_{m}}{8 C_{0}}, \\
& \mathrm{Q}=\frac{8}{\pi^{2} R_{m}} \sqrt{\frac{L_{m}}{C_{m}}},
\end{aligned}
$$

Antenna gain calculation. Through the gain-transfer (gain-comparison) method, the gain of SMR antennas at the resonance frequency $f_{0}$ is expressed as:

$$
G_{S M R}=G_{R}+S_{21, S M R}+S_{21, R}
$$

where $G_{R}$ is the gain of the reference antenna, $S_{21, S M R}$ and $S_{21, R}$ are the transmitting S parameters of SMR antenna and reference antenna. GR of the horn antenna is $10.6 \mathrm{dBi}$ at $1.75 \mathrm{GHz}$.

\section{Data availability}

All relevant data in the article are available from the corresponding author on reasonable request.

\section{References}

47. Lou J, Insignares R, Cai Z, Ziemer KS, Liu M, Sun NX. Soft magnetism, magnetostriction, and microwave properties of FeGaB thin films. Applied Physics Letters 91, 182504 (2007).

48. Liang X, et al. Soft Magnetism, Magnetostriction and Microwave Properties of Fe-Ga-C Alloy Films. IEEE Magnetics Letters, (2018).

49. Pearton S, Norton D, Ip K, Heo Y, Steiner T. Recent progress in processing and properties of ZnO. Progress in materials science 50, 293-340 (2005).

50. $\quad \mathrm{Fu} \mathrm{YQ}$, et al. Recent developments on $\mathrm{ZnO}$ films for acoustic wave based bio-sensing and microfluidic applications: a review. Sensors and Actuators B: Chemical 143, 606-619 (2010). 


\section{Acknowledgements}

3 The financial supports from the NSF TANMS ERC Award 1160504, W.M. Keck Foundation. Special thanks to Dr. Mohan

4 Sanghadasa at U.S. Army Combat Capabilities Development Command Aviation \& Missile Center for their support. Army SBIR program award \# W9113M-19-C-0063. The UIUC team acknowledges support from Office of Naval Research (ONR) MURI through grant N00014-16-1-2436. The Kiel team acknowledges support from the German German Research Foundation (Deutsche Forschungsgemeinschaft, DFG) through the Collaborative Research Centre CRC 1261 "Magnetoelectric Sensors: From Composite Materials to Biomagnetic Diagnostics". The picosecond acoustics experiments were carried out in the Materials Research Laboratory 9 Central Research Facilities, University of Illinois.

\section{Author contributions}

X.L., H.C., and N.S. made the preliminary experimental observations. X.L., H.C., N.S., and N.X.S. conceived and designed the experiments. X.L., H.C., and N.S. conducted the experiments and characterisations, analysed the data with the help of C.M., E.G.,

14 S.M., Y.W., C.D., M.Z., Y.H., M.S., Y.G., H.L., D.G.C., and J.M.. The first manuscript was drafted by X.L. and N.X.S. with inputs 15 from all the other authors.

\section{Competing interests}

18 The authors declare no competing financial interests.

\section{Additional information}

21 Supplementary information is available in the online version of the paper.

Correspondence and requests for materials should be addressed to Nian X. Sun.

23 Reprints and permissions information is available online at www.nature.com/reprints.

Publisher's note: Springer Nature remains neutral with regard to jurisdictional claims in published maps and institutional affiliations. 
Figures and tables

a

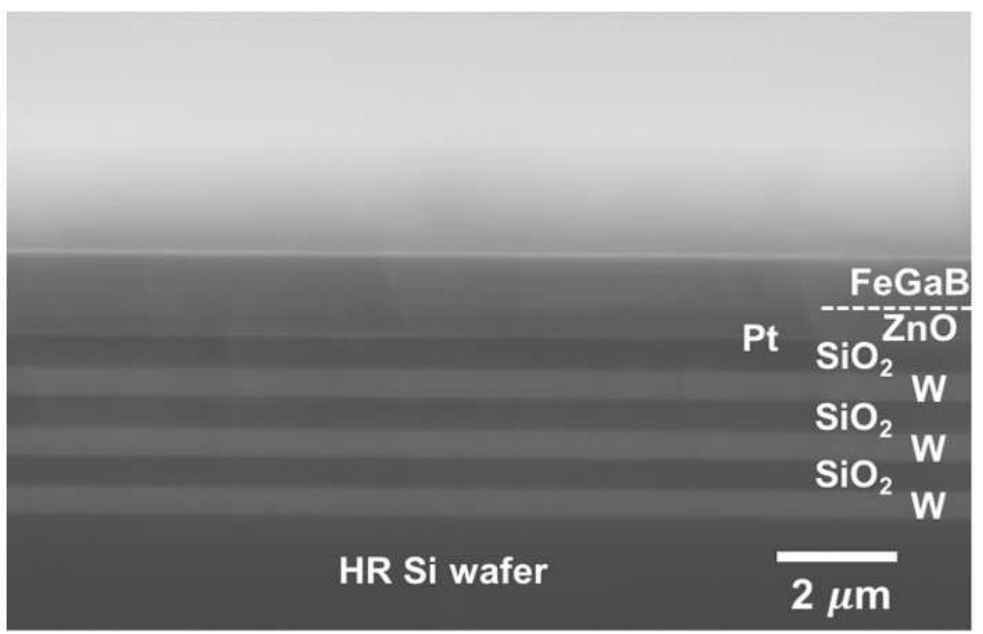

C
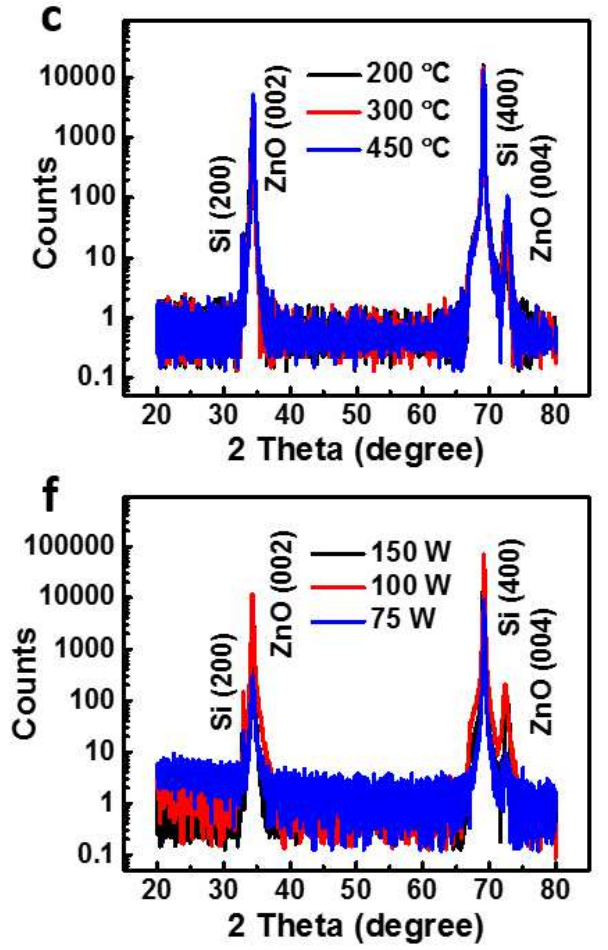

d b
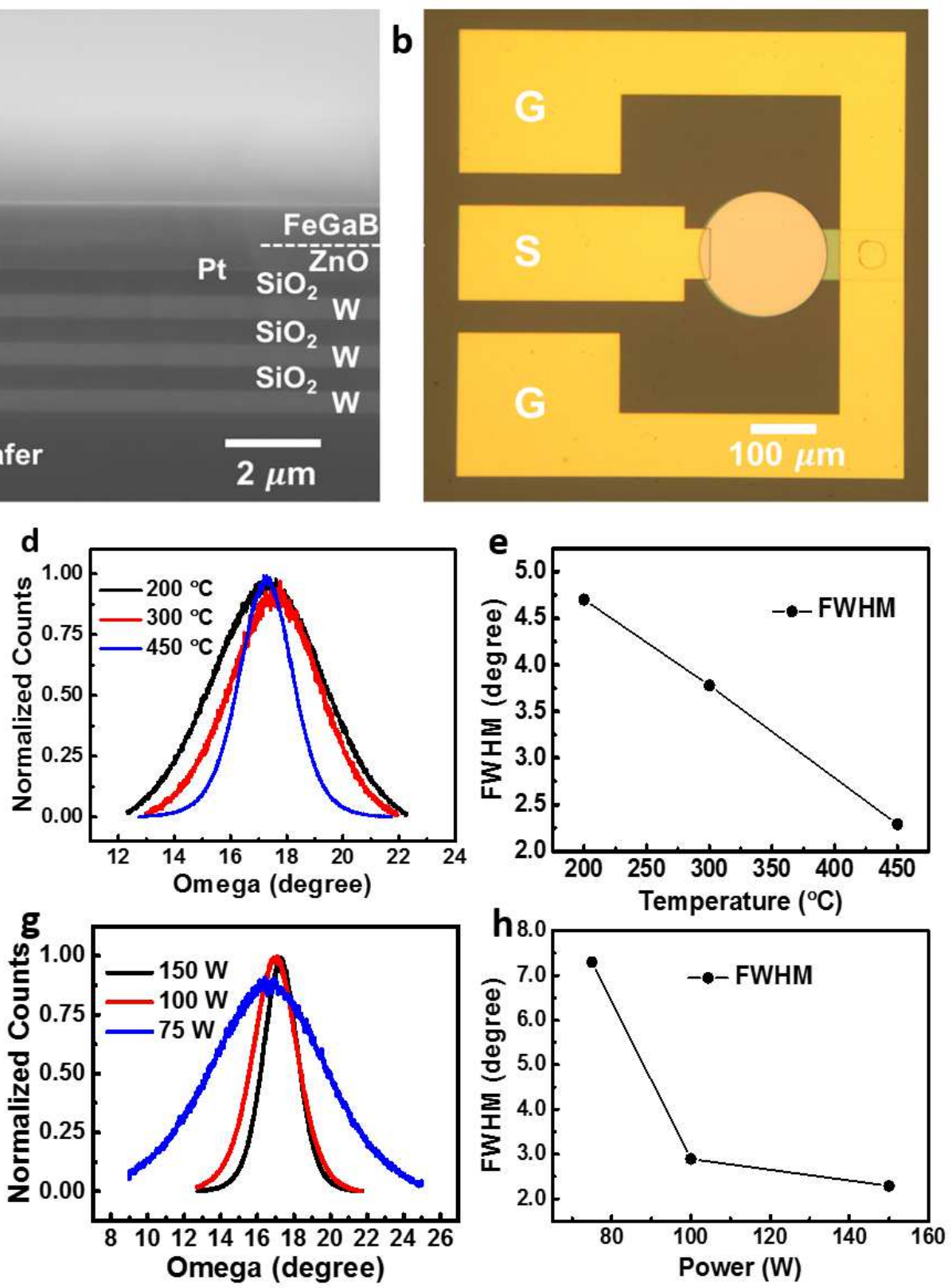

Fig. 1 | Structural properties of the fabricated SMR ME antenna. a, Cross-sectional SEM image of the ME antenna. b, Optical image of the ME antenna. Ground (G) and signal (S) pads are used to feed the RF signal into the device. $\mathbf{c}, \mathbf{f}, \mathrm{XRD}$ patterns of ZnO films with increasing deposition temperature and RF plasma power. $\mathbf{d}$, g, Rocking curves of $\mathrm{ZnO}$ films with increasing deposition temperature and RF plasma power. All curves are fitted with Gauss function to get the FWHM values. e, h, FWHM versus deposition temperature and plasma power. 

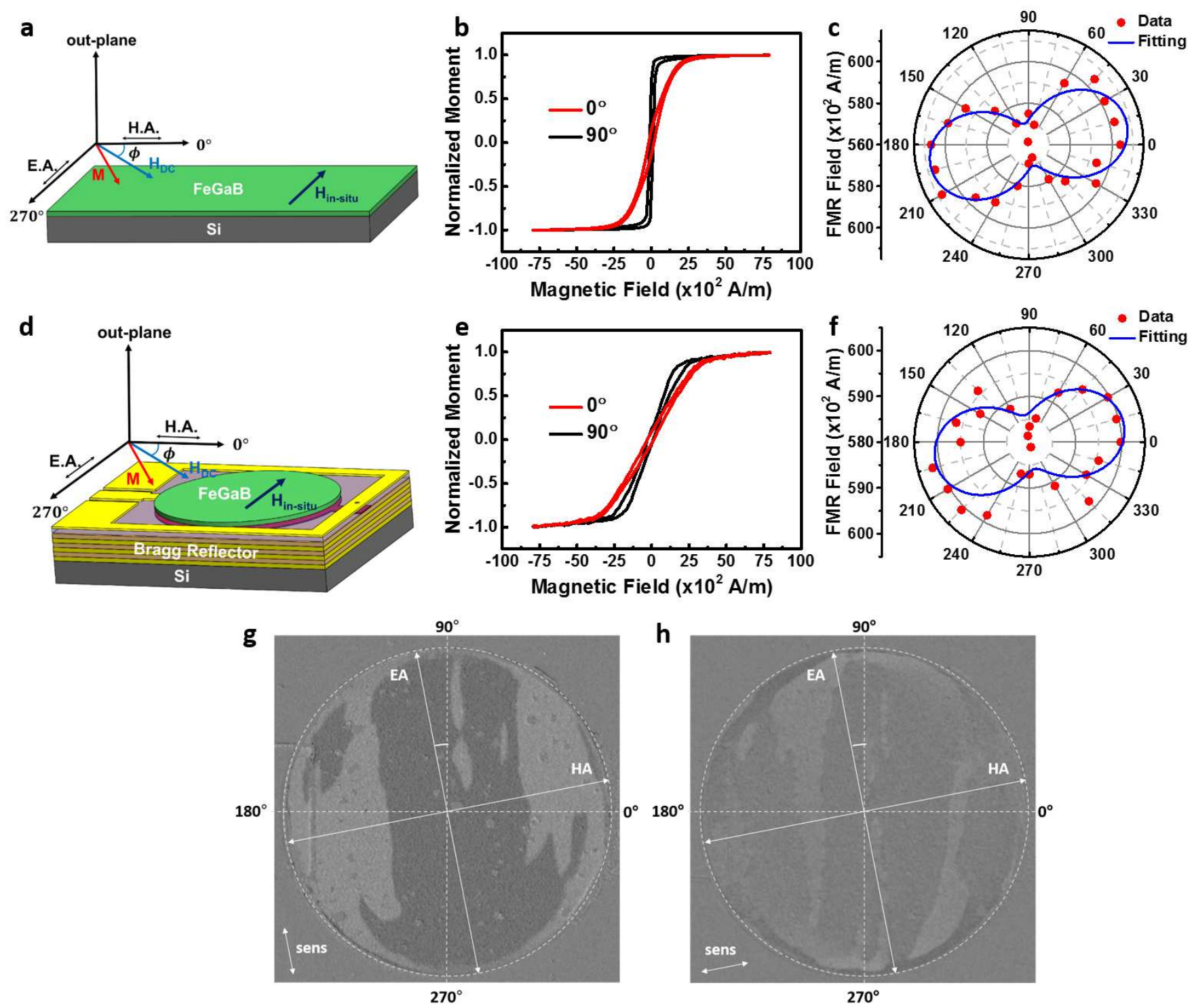

$270^{\circ}$

Fig. 2 | Magnetic properties of $\mathbf{F e G a B} / \mathrm{SiO}_{2}$ multilayers. a, d, Coordinate systems for in-plane analysis of the reference full film and patterned device. $0^{\circ}$ is aligned perpendicular to the in-situ magnetic field. $\mathbf{b}$, e, Magnetisation loops for the reference full film and the patterned device. Black: along the 90-degree axis; Red: along the 0-degree axis. $\mathbf{c}, \mathbf{f}$, In-plane angular dependence of FMR fields for the reference full film and patterned device. Red points: experimentally measured data; blue lines: fitted data. $\mathbf{g}$, $\mathbf{h}$ Remanent state after magnetic field application aligned along the hard axis of the sample (11-degree axis). MOKE sensitivity is along the $101^{\circ}$ (E.A.) (g) and $11^{\circ}$ (H.A.) (h) axis. 

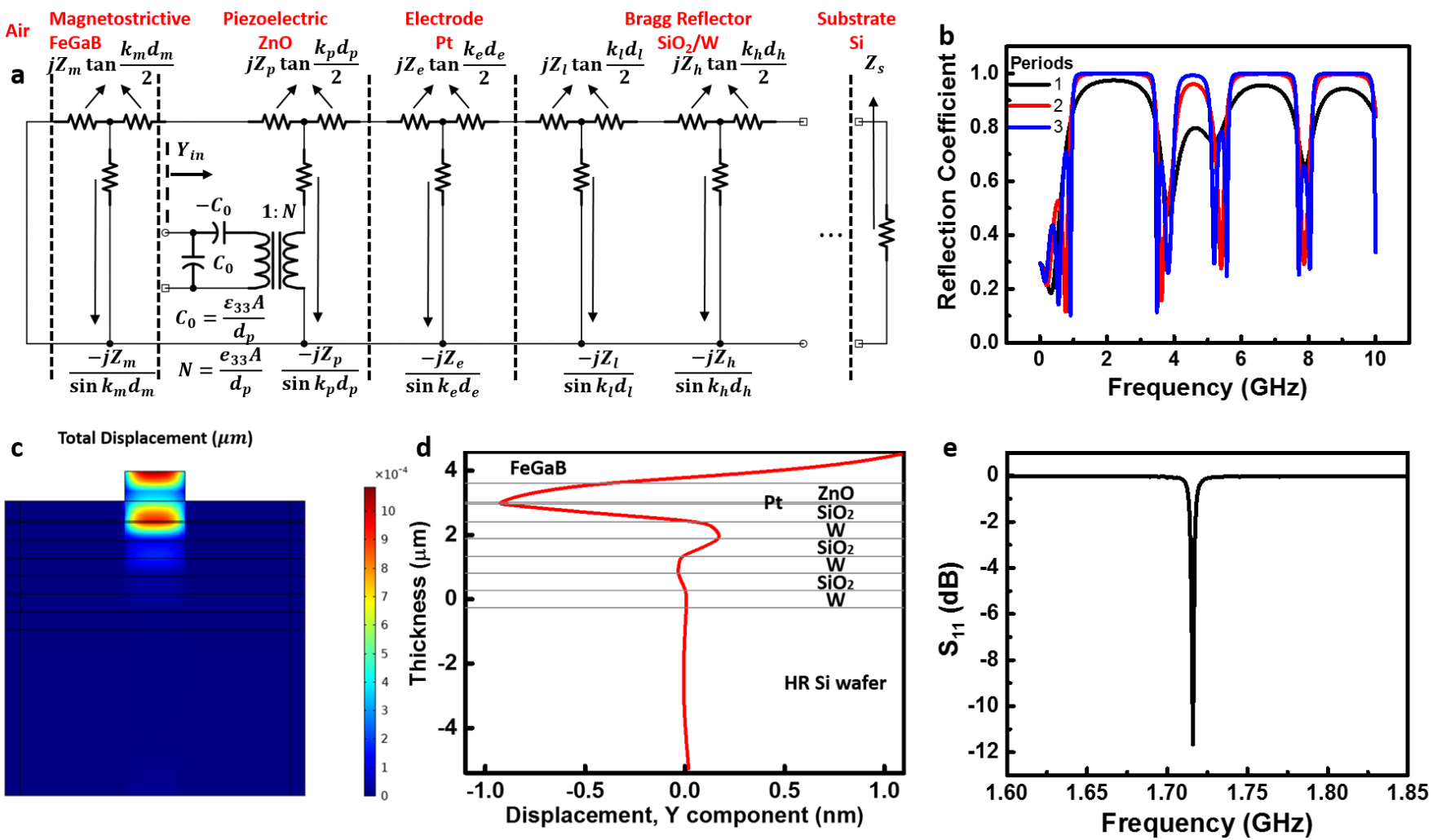

Fig. 3 | $1 D$ Mason model and 2D finite element model (FEM) COMSOL simulation results of the proposed SMR-based ME antenna. a, Equivalent circuit of the SMR-based ME antenna analysed by Mason's model. $Z_{i}$, $\mathrm{k}_{\mathrm{i}}$ and $\mathrm{d}_{\mathrm{i}}$ : acoustic impedance, propagation constant and thickness of each layer; $\mathrm{C}_{0}$ : static capacitance of the resonator; A: electrode area; $\varepsilon_{33}$ and $e_{33}$ are dielectric

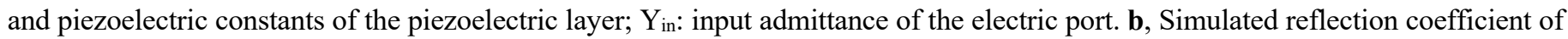
the designed Bragg reflector consisting of various pairs of $\mathrm{SiO}_{2} / \mathrm{W}$ layers based on the Mason model. c, Displacement profile at resonant frequency. d, Standing wave amplitude as a function of depth and e, Frequency response of the designed ME antenna simulated with the 2D FEM COMSOL model. 

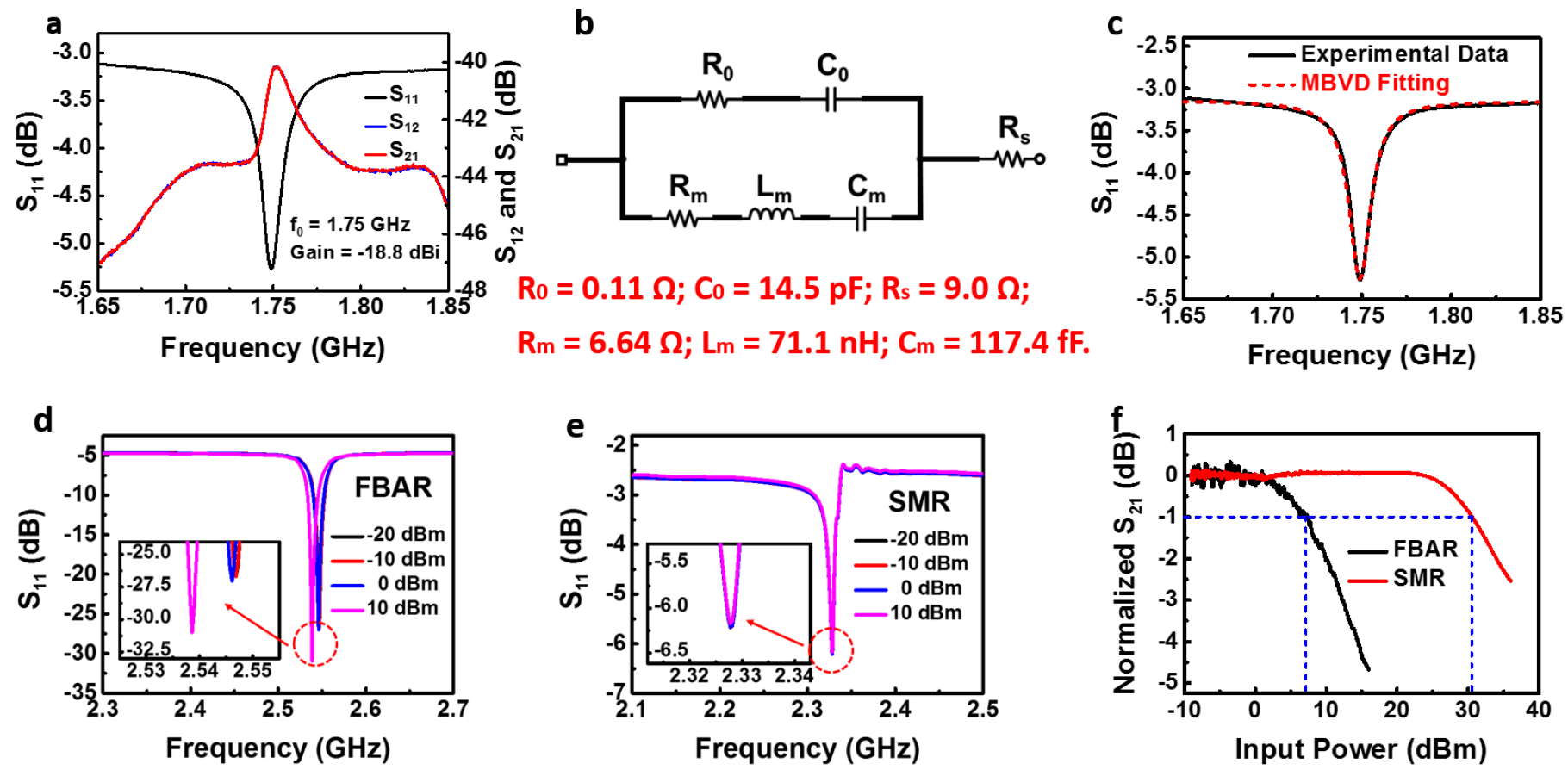

Fig. 4 | Modified Butterworth Van Dyke (MBVD) model and power handling measurements of the SMR antenna. a, Return loss curve $\left(\mathrm{S}_{11}\right)$, receiving $\left(\mathrm{S}_{12}\right)$ and transmitting behaviour $\left(\mathrm{S}_{21}\right)$ of the SMR antenna with resonant frequency of $1.75 \mathrm{GHz}$ and antenna gain of $-18.8 \mathrm{dBi}$. b, Schematics of MBVD model with fitted values. $\mathbf{c}$, Return loss curves $\left(\mathrm{S}_{11}\right)$ for experimental data and MBVD fitting. d, $\mathrm{S}_{11}$ results of the FBAR antenna at different input power. The resonance peak shifts to lower frequency with input power of $10 \mathrm{dBm}$, which means the FBAR antenna goes into nonlinear region. $\mathbf{e}, \mathrm{S}_{11}$ results of the SMR antenna at different input power. The antenna performance is stable without any frequency shift. f, Power sweep of the FBAR and SMR antennas. The FBAR antenna has a $1 \mathrm{~dB}$ compression point $(\mathrm{P} 1 \mathrm{~dB})$ of $7.1 \mathrm{dBm}$ while the $\mathrm{P} 1 \mathrm{~dB}$ of SMR antenna is $30.4 \mathrm{dBm}$, which shows the better power handling capability of the SMR antenna. 

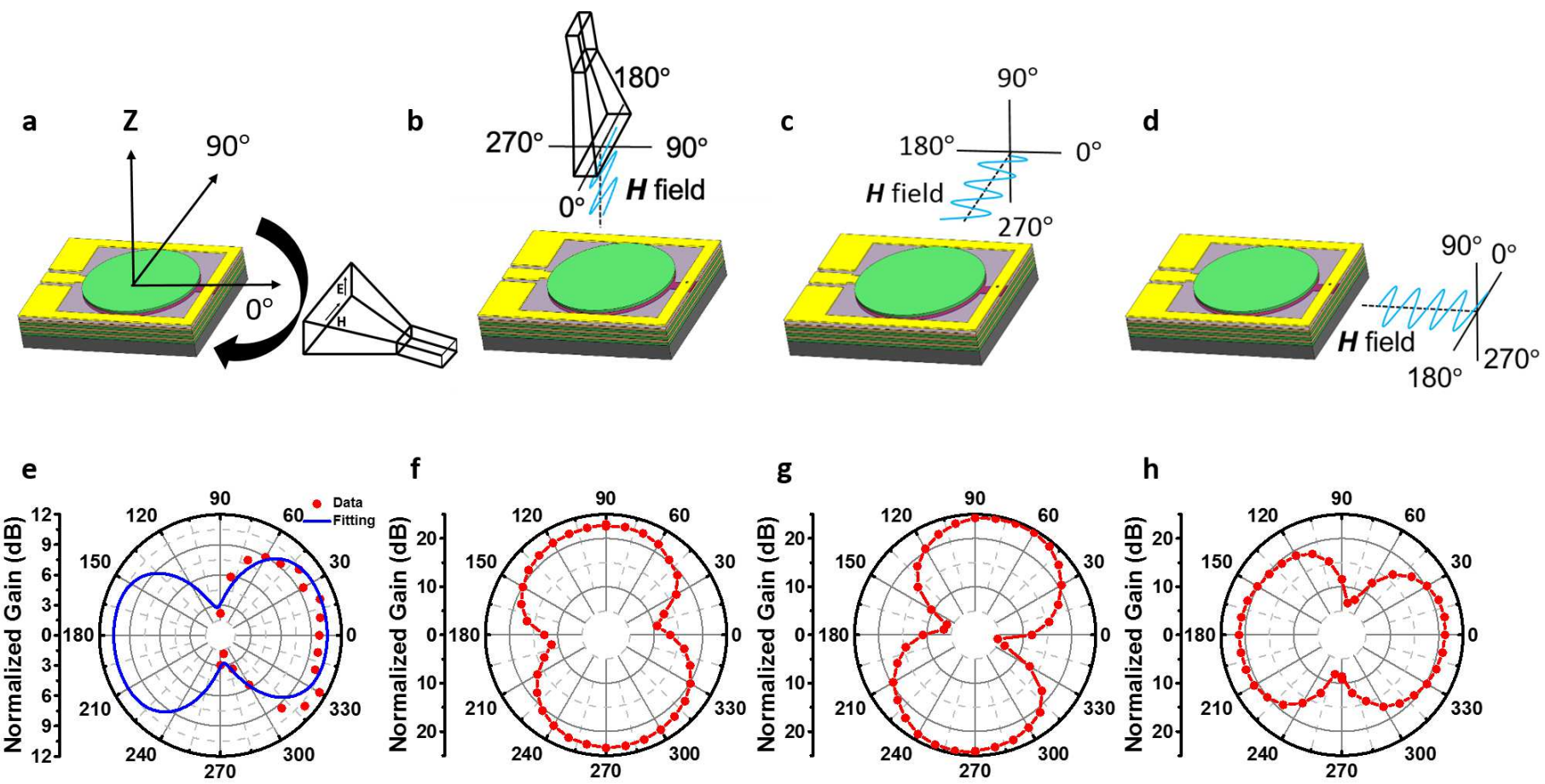

Fig. 5 | Antenna performance of the SMR device. a-d, Schematic representations of the test setups for the in-plane radiation pattern and polarisation along three major axes measurements under a calibrated horn antenna. e, In-plane radiation pattern. f-h, Normalised gain patterns of antenna polarisation for the in-plane rotation, out-of-plane rotation perpendicular the anchor direction and out-ofplane rotation along the anchor direction. The sinusoidal wave along $0^{\circ}$ and $180^{\circ}$ denotes the $\mathrm{H}$-field component of the EM waves from horn antenna. Red dots represent the experimental data; Blue line is the fitted curve of the cosine function; Red lines are guides for the eyes.

Table 1. Performance comparison between the FBAR and SMR ME antennas.

\begin{tabular}{lllllll}
\hline \hline ME & Frequency & $\mathbf{S}_{11}$ & Gain & P1dB & Process & Robustness \\
Antenna & $(\mathbf{G H z})$ & $\mathbf{( d B})$ & $\mathbf{( d B i )}$ & $\mathbf{( d B m )}$ & Control & \\
& & & & & & \\
\hline FBAR & 2.53 & $<-20$ & -18 & 7.1 & Hard & Bad \\
SMR & 1.75 & $\sim-5$ & -18.8 & 30.4 & Easy & Good \\
\hline \hline
\end{tabular}

9 


\section{Supplementary Files}

This is a list of supplementary files associated with this preprint. Click to download.

- SupplementaryNatureCommunications.pdf 